\title{
Interpolation solution of the single-impurity Anderson model
}

\author{
Alexander L. Kuzemsky ${ }^{1}$ \\ International Centre for Theoretical Physics, Trieste, Italy \\ Received 19 September 1990; accepted for publication 10 January 1991 \\ Communicated by A.A. Maradudin
}

\begin{abstract}
The dynamical properties of the single-impurity Anderson model (SIAM) is studied using a novel irreducible Green function method (IGF). A new solution for the one-particle GF, interpolating between the strong and weak correlation limits, is obtained. The unified concept of relevant mean-field renormalizations is indispensable for the strong correlation limit.
\end{abstract}

\section{Introduction}

The single-impurity Anderson model (SIAM) has been proposed many years ago [1] to describe highly dilute random alloys $[2,3]$. In practice, this model is used to imitate other systems too. For example more recently this model has been widely used to describe mixed valence and heavy fermion systems [4-7]. Although it is a simplified model, nevertheless it contains most of the relevant physics and a great deal of interesting work has been done on it [2-7]. But it still remains only partially solved. The elegant Bethe-ansatz technique as applied to the reduced Anderson model [3] leads to the exact static solution of SIAM only. The generalization of this approach for a realistic $k^{2}$ electron spectrum has not been done yet.

In the past years many efforts have been made to calculate dynamical properties of SIAM using various advanced methods of many-body theory [4-15]. Unfortunately, the proposed solutions are, as a rule, limited in several ways; they are valid for a rather narrow interval of relevant parameters. Moreover, no general concept for construction of the interpolating dynamical solution of SIAM has been proposed. In such a situation the unified self-consistent approach, which permits one to obtain a solution interpolating between the weak and strong correlation limits, is highly desirable.

In this paper we present a unified self-consistent calculation of the one-electron Green function(GF) which gives the correct results both for the weak and the strong Coulomb correlations. The approach we suggest is founded on the same type of concept which has proved to be valuable for the Hubbard model [16,17] and which has been suggested as essential for various many-body systems with complex spectrum and strong interaction [16-21]. We believe that the solutions which are derived below bear the real physics of SIAM.

\section{Outline of the method}

The irreducible GF (IGF) method allows one to describe completely the quasiparticle spectra with damping in a very general way. It is based on the notion of the "irreducible" parts of GFs (or the irreducible parts of the operators, out of which the GF is constructed) in terms of which it is possible, without recourse to a trun-

1 Permanent address and address for correspondence: Department of Statistical Mechanics, Laboratory of Theoretical Physics, Joint Institute for Nuclear Research (Dubna), Head Post Office P.O. Box 79, 101000 Moscow, USSR. 
cation of the hierarchy of equations for the GFs, to write down the exact Dyson equation and to obtain an exact analytical representation for the self-energy operator. Therefore, in contrast to the standard equation-ofmotion approach, the decoupling is introduced in the self-energy operator only. The general philosophy of the IGF method lies in the separation and identification of elastic scattering effects and inelastic ones. The irreducible GFs are so defined that they cannot be reduced to the lower-order ones by any way of decoupling. This procedure extracts all relevant (for the problem under consideration) mean-field contributions and puts them into the generalized mean-field GF. It is worth emphasizing that, in general, the mean-field renormalizations can exhibit a quite nontrivial structure. To obtain this structure correctly, one must construct the full GF built of the complete algebra of relevant operators and develop a special projection procedure for higher-order GFs in accordance with the algebra found. The most important feature of this approach is a very nontrivial structure of the mean-field renormalizations as found in the cases of the Hubbard model in the strong correlation limit [16] and the magnetic polaron problem at finite temperatures and an arbitrary value of s-f exchange $[19,20]$. It is important to note that there is a possibility of generalizing the scheme described above introducing IGFs for higher-order equations of motion [21].

\section{SIAM, weak correlation}

Let us consider the standard one-impurity Anderson Hamiltonian [1]:

$H=\sum_{k \sigma} \epsilon_{k} c_{k \sigma}^{+} c_{k \sigma}+\epsilon_{d} \sum_{\sigma} d_{0 \sigma}^{+} d_{0 \sigma}+\frac{1}{2} U \sum_{\sigma} n_{0 \sigma}^{d} n_{0-\sigma}^{d}+\sum_{k \sigma} V_{k}\left(c_{k \sigma}^{+} d_{0 \sigma}+d_{0 \sigma}^{+} c_{k \sigma}\right)$.

This is the basic Hamiltonian which will be discussed here. The simplest way of dealing with (1) is to apply the Hartree-Fock (HF) theory [1]. But, as was pointed out in the review paper [3], it is a fairly rough approximation which has no region of applicability. Nevertheless, from the formal point of view we consider that the validity region of it is limited to weak correlation $(U \rightarrow 0)$ only. In this limit, due to the mixing of $d$ and $s$ states, the resonance level exhibits a finite width. We will give below the IGF calculations for $U \rightarrow 0$.

For the calculation of the electronic quasiparticle spectrum of the model described by the Hamiltonian (1) let us consider the equations of motion for one-electron GFs

$$
\begin{aligned}
& \left\langle\left\langle c_{k \sigma}(t) c_{k \sigma}^{+}\left(t^{\prime}\right)\right\rangle=-\mathrm{i} \theta\left(t-t^{\prime}\right)\left\langle\left[c_{k \sigma}(t), c_{k \sigma}^{+}\left(t^{\prime}\right)\right]_{+}\right\rangle,\right. \\
& \left\langle\left\langle d_{0 \sigma}(t) d_{0 \sigma}^{+}\left(t^{\prime}\right)\right\rangle=-\mathrm{i} \theta\left(t-t^{\prime}\right)\left\langle\left[d_{0 \sigma}(t), d_{0 \sigma}^{+}\left(t^{\prime}\right)\right]_{+}\right\rangle .\right.
\end{aligned}
$$

Because of $c-d$ coupling the GFs (2) and (3) are elements of the matrix GF,

$$
\hat{G}(\omega)=\left(\begin{array}{ll}
\left\langle c_{k \sigma} \mid c_{k \sigma}^{+}\right\rangle_{\omega} & \left\langle\left\langle c_{k \sigma} \mid d_{0 \sigma}^{+}\right\rangle_{\omega}\right. \\
\left\langle\left\langle d_{0 \sigma} \mid c_{k \sigma}^{+}\right\rangle_{\omega}\right. & \left\langle\left\langle d_{0 \sigma} \mid d_{0 \sigma}^{+}\right\rangle_{\omega}\right.
\end{array}\right)
$$

Performing first time $t$ differentiation of (4) and introducing the irreducible GF by the definition [16,17]

${ }^{\text {ir }}\left\langle\left\langle d_{0 \sigma} n_{0-\sigma} \mid d_{0 \sigma}^{+}\right\rangle_{\omega}=\left\langle\left\langle d_{0 \sigma} n_{0-\sigma} \mid d_{0 \sigma}^{+}\right\rangle_{\omega}-\left\langle n_{0-\sigma}\right\rangle\left\langle\left\langle d_{0 \sigma} \mid d_{0 \sigma}^{+}\right\rangle_{\omega}\right.\right.\right.$,

we obtain the following equation in matrix form,

$\sum_{p} \Phi_{p}(\omega) \hat{G}(\omega, p)=\hat{1}+U \hat{D}^{\mathrm{ir}}(\omega)$

where all definitions are rather evident. In order to calculate the higher-order GF on the r.h.s. of (6) we have to write the equation of motion obtained by means of differentiation with respect to the second time variable $t^{\prime}$. Definition (5) allows us to remove the inhomogeneous term in this equation. If one introduces irreducible parts for the r.h.s. operators by analogy with expression (5), the equation of motion (6) can be exactly rewritten in the form of the Dyson equation 
$\hat{G}=\hat{G}^{0}+\hat{G}^{0} \hat{M} \hat{G}$.

The generalized mean-field GF $\hat{G}^{0}$ satisfies the equation

$\sum_{p} \Phi_{p}(\omega) \hat{G}^{0}(\omega, p)=\hat{1}$.

The explicit solution of (8) for diagonal elements is

$\left\langle\left\langle c_{k \sigma} \mid c_{k \sigma}^{+}\right\rangle_{\omega}^{0}=\left(\omega-\epsilon_{k}-\frac{\left|V_{k}\right|^{2}}{\omega-\epsilon_{d}-U n_{-\sigma}}\right)^{-1}\right.$,

$\left\langle d_{0 \sigma} \mid d_{0 \sigma}^{+}\right\rangle{ }_{\omega}^{0}=\left(\omega-\epsilon_{d}-U n_{-\sigma}-\sum_{p} \frac{\left|\underline{K}_{p}\right|^{2}}{\omega-\epsilon_{p}}\right)^{-1}$.

These expressions coincide with Anderson's HF result [1] and in the limit $U=0$ lead to exact results for GFs.

The self-energy operator $M$, which describes inelastic scattering processes, has the following matrix form:

$\hat{M}=\left(\begin{array}{cc}0 & 0 \\ 0 & M_{00}^{\sigma}\end{array}\right)$,

where

$M_{00}^{\sigma}=U^{2}\left\langle\left\langle d_{0 \sigma} n_{0-\sigma} \mid d_{0 \sigma}^{+} n_{0-\sigma}\right\rangle\right.$.

Thus, by introducing irreducible parts of GF (or the irreducible parts of the operators, out of which the GF is constructed) the equations of motion for the GF (4) were exactly transformed into the Dyson equation (7) with an exact representation of the self-energy operator, expressed in terms of higher order GF. It should be emphasized that for the weak correlation case, $U \rightarrow 0$, the functional of the mean-field renormalization can be represented in terms of mean densities of electrons (see eq. (5)).

The formal solution of the Dyson equation (7) can be written as

$\hat{G}=\left[\left(\hat{G}^{0}\right)^{-1}-\hat{M}\right]^{-1}$.

From (13) one immediately obtains

$$
\begin{aligned}
& \left\langle\left\langle d_{0 \sigma} \mid d_{0 \sigma}^{+}\right\rangle_{\omega}=\left(\omega-\epsilon_{d}-U n_{-\sigma}-M_{00}^{\sigma}-\sum_{p} \frac{\left|V_{p}\right|^{2}}{\omega-\epsilon_{p}}\right)^{-1},\right. \\
& \left\langle\left\langle c_{k \sigma} \mid c_{k \sigma}^{+}\right\rangle_{\omega}=\left(\omega-\epsilon_{k}-\frac{\left|V_{k}\right|^{2}}{\omega-\epsilon_{d}-U n_{-\sigma}-M_{00}^{\sigma}}\right)^{-1} .\right.
\end{aligned}
$$

In order to calculate the self-energy operator in.a self-consistent way, we have to express it approximately by lower order GFs. Let us start in analogy with the Hubbard model [16] with a pair-type approximation

$$
\begin{aligned}
& M_{00}^{\sigma}(\omega) \approx U^{2} \int_{-\infty}^{\infty} \frac{\mathrm{d} \omega_{1} \mathrm{~d} \omega_{2} \mathrm{~d} \omega_{3}}{\omega+\omega_{1}-\omega_{2}-\omega_{3}}\left\{n\left(\omega_{1}\right)\left[1-n\left(\omega_{2}\right)-n\left(\omega_{3}\right)\right]\right. \\
& \left.+n\left(\omega_{2}\right) n\left(\omega_{3}\right)\right\} g_{0-\sigma}\left(\omega_{1}\right) g_{0 \sigma}\left(\omega_{2}\right) g_{0-\sigma}\left(\omega_{3}\right),
\end{aligned}
$$

where

$g_{0 \sigma}(\omega)=-\frac{1}{\pi} \operatorname{Im}\left\langle\left\langle d_{0 \sigma} \mid d_{0 \sigma}^{+}\right\rangle_{\omega}\right.$.

If we take for the first iteration step 
$g_{0 \sigma}(\omega) \sim \delta\left(\omega-\epsilon_{d}-U n_{-\sigma}\right)$,

we immediately obtain $M_{00}^{\sigma}=0$. This result reflects the fact that only one impurity site is present. For the periodic Anderson model the pair approximation (15) should work quite well.

Let us try (again in analogy with the Hubbard model [16]) another type of approximation for $M$. Owing to the well known spectral theorem the GF in the r.h.s. of (12) can be expressed in terms of correlation functions. The approximation which we will use now reflects the interference between the one-particle branch and the collective one:

$$
\begin{aligned}
& \left\langle d_{0-\sigma}^{+} d_{0-\sigma} d_{0 \sigma}^{+} d_{0 \sigma}(t) d_{0-\sigma}^{+}(t) d_{0-\sigma}(t)\right\rangle \approx\left\langle n_{0-\sigma} n_{0-\sigma}(t)\right\rangle\left\langle d_{0 \sigma}^{+} d_{0 \sigma}(t)\right\rangle \\
& \quad+\left\langle d_{0 \sigma}^{+} d_{0-\sigma} d_{0-\sigma}^{+}(t) d_{0 \sigma}(t)\right\rangle\left\langle d_{0-\sigma}^{+} d_{0-\sigma}(t)\right\rangle+\left\langle d_{0 \sigma}^{+} d_{0-\sigma}^{+} d_{0-\sigma}(t) d_{0 \sigma}(t)\right\rangle\left\langle d_{0-\sigma} d_{0-\sigma}^{+}(t)\right\rangle .
\end{aligned}
$$

If we retain only the first term in (17) (cf. ref. [12]) and make use of the same first iteration as (16), we obtain for the self-energy operator

$$
M_{00}^{\sigma}(\omega) \simeq U^{2} \frac{1-n\left(\epsilon_{d}-U n_{-\sigma}\right)}{\omega-\epsilon_{d}-U n_{-\sigma}}\left\langle n_{0-\sigma} n_{0-\sigma}\right\rangle
$$

It is very interesting that when retaining the second term in (17) we obtain

$$
\begin{aligned}
& \left\langle d_{0 \uparrow} \mid d_{0 \uparrow}^{+}\right\rangle_{\omega}=\left(\omega-\epsilon_{d}-U n_{\downarrow}-M_{00}^{\dagger}(\omega)-\sum_{p} \frac{\left|\underline{V}_{p}\right|^{2}}{\omega-\epsilon_{p}}\right)^{-1}, \\
& \left\langle\left\langle d_{0 \downarrow} \mid d_{0 \downarrow}^{+}\right\rangle_{\omega}=\left(\omega-\epsilon_{d}-U n_{\uparrow}-M_{00}^{\dagger}(\omega)-\sum_{p} \frac{\left|V_{p}\right|^{2}}{\omega-\epsilon_{p}}\right)^{-1},\right.
\end{aligned}
$$

where

$M_{00}^{\sigma}(\omega)=U^{2} \int_{-\infty}^{\infty} \mathrm{d} \omega_{1} \mathrm{~d} \omega_{2} \frac{1+N\left(\omega_{1}\right)-n\left(\omega_{2}\right)}{\omega-\omega_{1}-\omega_{2}}\left(-\frac{1}{\pi} \operatorname{Im}\left\langle\left\langle S_{0}^{\mp} \mid S_{0}^{ \pm}\right\rangle\right\rangle_{\omega_{1}}\right)\left(-\frac{1}{\pi} \operatorname{Im}\left\langle\left\langle d_{0 \sigma} \mid d_{0 \sigma}^{+}\right\rangle_{\omega n}\right)\right.$,

with $S_{0}^{+}=d_{0 \uparrow}^{+} d_{0 \downarrow}$ and $S_{0}^{-}=d_{0 \downarrow}^{+} d_{0 \uparrow}$, or, in more convenient form (cf. ref. [12]),

$M_{00}^{\sigma}(\omega)=U^{2} \int \mathrm{d} \omega_{1}\left(\operatorname{coth} \frac{\omega-\omega_{1}}{2 T}+\tanh \frac{\omega_{1}}{2 T}\right)\left(-\frac{1}{\pi} \operatorname{Im} \chi_{00}^{\mp \pm}\left(\omega-\omega_{1}\right)\right) g_{0 \sigma}\left(\omega_{1}\right)$.

The essential feature of this approximation is connected with the fact that spin up and spin down electrons are correlated when they occupy the impurity level. So this really improves the HF theory in which just these correlations are missed. The scattering of the d-electron with the band electron causes the impurity level to be shifted and broadened. But by including the correlation effects for the weak correlation case we obtain additional shift and broadening due to electron-electron inelastic scattering processes. This, of course, leads to small corrections for shift and width and influence mainly the line form of the spectral density. The role of electron-electron correlation becomes much more crucial for the case of strong correlation. In the region where $U$ is very large, but finite, the theory faces the most serious difficulties [14].

\section{SIAM, strong correlation}

To depict the behavior of the system in the case of strong Coulomb correlations when the scattering between the band and $d$ electrons is present we need a more sophisticated approach than the one proposed in refs. [815 ]. The relevant algebra of the operators used for the description of the strong correlation has a similar form 
as for the Hubbard model [16]. Let us represent the matrix GF (4) in the following form,

$\hat{G}_{\sigma}(\omega)=\left(\begin{array}{cc}\left\langle\left\langle c_{k \sigma} \mid c_{k \sigma}^{+}\right\rangle_{\omega}\right. & \sum_{\beta}\left\langle\left\langle c_{k \sigma} \mid f_{0 \beta \sigma}^{+}\right\rangle_{\omega}\right. \\ \sum_{\alpha}\left\langle\left\langle f_{0 \alpha \sigma} \mid c_{k \sigma}^{+}\right\rangle_{\omega}\right. & \sum_{\alpha \beta}\left\langle\left\langle f_{0 \alpha \sigma} \mid f_{0 \beta \sigma}^{+}\right\rangle_{\omega}\right.\end{array}\right)$,

where

$f_{0 \alpha \sigma}=n_{0-\sigma}^{\alpha} d_{0 \sigma} \quad(\alpha= \pm) ; \quad n_{0 \sigma}^{+}=n_{0 \sigma}, \quad n_{\overline{0 \sigma}}^{-}=1-n_{0 \sigma}$.

The equation of motion for the auxiliary GF $\hat{\tilde{G}}$,

$\hat{G}_{\sigma}(\omega)=\left(\begin{array}{ccc}\left\langle\left\langle c_{k \sigma} \mid c_{k \sigma}^{+}\right\rangle_{\omega}\right. & \left\langle\left\langle c_{k \sigma} \mid f_{0+\sigma}^{+}\right\rangle_{\omega}\right. & \left\langle\left\langle c_{k \sigma} \mid f_{0-\sigma}^{+}\right\rangle_{\omega}\right. \\ \left\langle\left\langle f_{0+\sigma} \mid c_{k \sigma}^{+}\right\rangle_{\omega}\right. & \left\langle\left\langle f_{0+\sigma} \mid f_{0+\sigma}^{+}\right\rangle_{\omega}\right. & \left\langle\left\langle f_{0+\sigma} \mid f_{0-\sigma}^{+}\right\rangle_{\omega}\right. \\ \left\langle\left\langle f_{0-\sigma} \mid c_{k \sigma}^{+}\right\rangle_{\omega}\right. & \left\langle\left\langle f_{0-\sigma} \mid f_{0+\sigma}^{+}\right\rangle_{\omega}\right. & \left\langle\left\langle f_{0-\sigma} \mid f_{0-\sigma}^{+}\right\rangle_{\omega}\right.\end{array}\right)$,

in matrix notation reads

$\hat{E} \hat{G}_{\sigma}(\omega)-\hat{I}=\hat{D}$,

where

$\hat{E}=\left(\begin{array}{ccc}\omega-\epsilon_{k} & -V_{k} & -V_{k} \\ 0 & \omega-\epsilon_{d}-U_{+} & 0 \\ 0 & 0 & \omega-\epsilon_{d}-U_{-}\end{array}\right), \quad \begin{array}{rlr}U_{\alpha}=U, & \alpha=+, \\ & =0, & \alpha=-,\end{array}$

and

$\hat{I}=\left(\begin{array}{ccc}1 & 0 & 0 \\ 0 & n_{0-\sigma}^{+} & 0 \\ 0 & 0 & n_{0-\sigma}^{-}\end{array}\right), \quad \hat{D}=\left(\begin{array}{ccc}0 & 0 & 0 \\ D_{21} & D_{22} & D_{23} \\ D_{31} & D_{32} & D_{33}\end{array}\right)$

Here $\hat{D}$ is a higher-order GF. As an example we give now two matrix elements

$D_{22}=\left\langle\left\langle\left(c_{p \sigma} n_{0-\sigma}+d_{0-\sigma}^{+} c_{p-\sigma} d_{0 \sigma}-c_{p-\sigma}^{+} d_{0-\sigma} d_{0 \sigma}\right) \mid f_{0+\sigma}^{+}\right\rangle_{\omega}\right.$,

$D_{33}=\left\langle\left\langle\left(c_{p \sigma}\left(1-n_{0-\sigma}\right)-d_{0-\sigma}^{+} c_{p-\sigma} d_{0 \sigma}+c_{p-\sigma}^{+} d_{0-\sigma} d_{0 \sigma}\right) \mid f_{0-\sigma}^{+}\right\rangle_{\omega}\right.$.

Let us introduce the matrix irreducible GF $\hat{D}^{\text {ir }}$ in accordance with the definition given in ref. [16],

$\hat{D}^{\mathrm{ir}}=\hat{D}-\sum_{\alpha}\left(\begin{array}{l}A^{+\alpha} \\ A^{-\alpha}\end{array}\right)\left(\begin{array}{ll}\tilde{G}_{\sigma}^{\alpha+} & \tilde{G}_{\sigma}^{\alpha-}\end{array}\right)$,

where the coefficients $A^{\alpha \beta}$ are determined from the condition

$\left\langle\left[\hat{D}_{\alpha \beta}^{\mathrm{ir}}, f_{0 \beta \sigma}^{+}\right]_{+}\right\rangle \equiv 0$.

The corresponding exact Dyson equation is

$\hat{\tilde{G}}=\hat{\tilde{G}}^{0}+\hat{\tilde{G}}^{0} \hat{M} \hat{G}$,

where $\hat{G}^{0}$ is the generalised mean-field GF. It is very important to show the explicit form of the mean-field renormalizations,

$A^{++}=\frac{\left\langle\left(d_{0-\sigma}^{+} c_{p-\sigma}+c_{p-\sigma}^{+} d_{0-\sigma}\right)\left(n_{0 \sigma}-n_{0-\sigma}\right)\right\rangle}{\left\langle n_{0-\sigma}\right\rangle}$, 
$A^{--}=\frac{-\left\langle\left(d_{0-\sigma}^{+} c_{p-\sigma}+c_{p-\sigma}^{+} d_{0-\sigma}\right)\left(1+n_{0 \sigma}-n_{0-\sigma}\right)\right\rangle}{1-n_{0-\sigma}}$,

$A^{-+}=-A^{++}, A^{+-}=-A^{--}$.

The generalized mean-field GF of the d-electrons has the form

$$
\begin{gathered}
\left\langle\left\langle d_{0 \sigma} \mid d_{0 \sigma}^{+}\right\rangle\right\rangle_{\omega}^{0}=\frac{\left\langle n_{0-\sigma}\right\rangle}{\omega-\epsilon_{d}-U_{+}-\sum_{p} V_{p} A^{++}}\left(1+\frac{\sum_{p} V_{p} A^{-+}}{\omega-\overline{\epsilon_{d}}-U_{-}}\right) \\
+\frac{1-\left\langle n_{0-\sigma}\right\rangle}{\omega-\epsilon_{d}-U_{-}-\sum_{p} V_{p} A^{--}}\left(1+\frac{\sum_{p} V_{p} A^{+-}}{\omega-\epsilon_{d}-U_{+}}\right) .
\end{gathered}
$$

For $V_{p}=0$ we obtain the exact atomic solution

$F^{\mathrm{at}}=\frac{\left\langle n_{0-\sigma}\right\rangle}{\omega-\epsilon_{d}-U_{+}}+\frac{1-\left\langle n_{0-\sigma}\right\rangle}{\omega-\epsilon_{d}-U_{-}}$.

The conduction electron GF in this approximation reads

$$
\left\langle\left\langle c_{k \sigma} \mid c_{k \sigma}^{+}\right\rangle\right\rangle_{\omega}^{0}=\left[\omega-\epsilon_{k}-\left|V_{k}\right|^{2} F^{\text {at }}(\omega)\right]^{-1} \text {. }
$$

This form of solution also gives the correct expression for $V_{k}=0$. The self-energy operator has the form

$\hat{M}=\hat{I}^{-1}\left(\sum_{p q} V_{p} V_{q} \ll \mathscr{L}^{\mathrm{ir}}\left|\left(\mathscr{L}^{+}\right)^{\mathrm{ir}}\right\rangle\right) \hat{I}^{-1}$

This equation is an analogue of the equation for the self-energy operator in the Hubbard model [16] so we are not going to write it explicitly here especially because of its complicated structure. But it is important to note that the self-energy operator for the periodic Anderson model is much more similar to the self-energy operator of the Hubbard model.

\section{Conclusions}

In summary, we have obtained a new interpolation solution - the one-particle GF for the SIAM in the framework of the IGF formalism. In the weak correlation case the functional of the generalized mean-fields (GMF) depends only on the mean electron densities and this solution improves the HF solution [1] and allows one to incorporate the correlation of the spin-up and spin-down electrons occupying the impurity level, in a selfconsistent way. For the case of strong Coulomb correlation we obtained an essentially new solution confirming the statement [16-21] that in this case the mean-field renormalisations have a quite nontrivial structure and cannot be reduced to the mean density functional. The theory we suggest allows one to find explicitly the damping of quasiparticle excitations in a self-consistent way as was demonstrated in detail here for the weak correlation case. And lastly, it should be emphasized that SIAM and the periodic Anderson model (PAM) will have a very different structure from GMF as well as from the structure of inelastic scattering corrections due to the self-energy operator. A more detailed analysis of the obtained solution and a comparison with the solution for PAM will be published elsewhere.

\section{Acknowledgement}

The author would like to thank Professor Abdus Salam, the International Atomic Energy Agency and Unesco 
for hospitality at the International Centre for Theoretical Physics, Trieste, where this work has been done. I wish to express my gratitude to Professor A. Holas who carefully read the manuscript and made a number of very helpful comments and suggestions.

\section{References}

[1] P.W. Anderson, Phys. Rev. 124 (1961) 41.

[2] K.H. Fischer, Phys. Rep. 47 (1978) 225.

[3] A.M. Tsvelick and P.B. Wiegmann, Adv. Phys. 32 (1983) 453.

[4] H. Keiter and G. Morandi, Phys. Rep. 109 (1984) 227.

[5] G. Czycholl, Phys. Rep. 1343 (1986) 227.

[6] D.M. Newns and N. Read, Adv. Phys. 36 (1987) 799.

[7] P. Fulde, J. Keller and G. Zwicknagel, in: Solid state physics, eds. F. Seitz et al. (Academic Press, New York, 1988) p. 1.

[8] J.R. Schrieffer and D. Mattis, Phys. Rev. 140 (1965) A1412.

[9] A.C. Hewson, Phys. Rev. 144 (1966) 420.

[10] V. Zlatic, G. Gruner and N. Rivier, Solid State Commun. 14 (1974) 639.

[11] C. Lacroix, J. Phys. F 11 (1981) 2389.

[12] M. Salomaa, Solid State Commun. 38 (1981) 815.

[13] M. Salomaa, Solid State Commun. 39 (1981) 1105.

[14] Th. Pruschke and N. Grewe, Z. Phys. B 74 ( 1989) 439.

[15] G. Zwicknagel, V. Zevin and P. Fulde, Z. Phys. B 79 (1990) 365.

[16] A.L. Kuzemsky, Teor. Mat. Fiz. 36 (1978) 208 [Sov. Phys. Theor. Math. Phys. 36 (1978) 692 ].

[17] A.L. Kuzemsky, Dokl. Akad. Nauk SSSR 309 (1989) 323.

[18] D. Marvakov, J. Vlahov and A.L. Kuzemsky, J. Phys. C 18 (1985) 2871.

[19] D. Marvakov, A.L. Kuzemsky and J. Vlahov, Phys. Lett. A 105 (1984) 431.

[20] D. Marvakov, A.L. Kuzemsky and J. Vlahov, Physica B 138 (1986) 129.

[21 ] A.L. Kuzemsky, in: Proc. V Int. Symp. on Selected topics in statistical mechanics, eds. V.G. Kadyshevsky et al. (World Scientific, Singapore, 1990). 